\title{
Optimization of Extraction Technology of Flavonoids from Grapefruit by Orthogonal Test
}

\author{
Xian Qiu, Jing Wang, Minyi Lu, Yongguang $\mathrm{Bi}^{\mathrm{a} *}$ \\ College of Pharmacy, Guangdong Pharmaceutical University, Guangzhou 510006, Guangdong, \\ China \\ abiyongguang2002@163.com \\ ${ }^{*}$ Corresponding author:
}

Keywords: Grapefruit Seed; Flavonoids; Orthogonal Test Method

\begin{abstract}
In this paper, the technology of ultrasonic extraction of flavonoids from grapefruit seed was studied. The orthogonal design method was used to optimize the process. The results showed that the optimum extraction parameters were ultrasonic ratio 1:50 $(\mathrm{g} / \mathrm{mL})$, Ultrasonic power of $280 \mathrm{w}$, extraction temperature $65{ }^{\circ} \mathrm{C}$, extraction time $30 \mathrm{~min}$. Under these conditions, the extraction rate of flavonoids from grapefruit seed was $3.380 \mathrm{mg} / \mathrm{g}$. Ultrasonic enhanced extraction has the advantages of low temperature, high extraction efficiency and low cost, which can provide reference for industrial application.
\end{abstract}

\section{Introduction}

The grapefruit is a genus of Rutaceae, which is widely distributed in the south bank of the Yangtze River in China. At present, Southeast Asia and its Americas region is also widely cultivated. Chinese medicine, theory that grapefruit cold, sour, rational gas phlegm, can eliminate the middle of the plot food sobering treatment of inflammation and other effects. Liang et al. Studied the contents of the exocarp in the grapefruit and the volatile oil. The inner peel contains a lot of polysaccharides and flavonoids. Grapefruit seed contains yellow ketone, cypressin cool, etc., studies have shown that grapefruit seed can play a role in repairing cells, for pigmentation and other melanin deposition have a certain effect. Grapefruit can enhance the body of the f beggar iron absorption, for women can prevent the occurrence of anemia ${ }^{[1]}$. The chemical composition of grapefruit seed meal was analyzed. The results showed that the crude fat content of grapefruit was about $40.21 \%$, and the yield of grapefruit seed oil was $18.04 \%$ by Soxhlet extraction method. At present, there are some studies on its fatty acid composition at home and abroad. It can be used as reference in this experiment. The fatty acids contained in grapefruit seed oil are palmitic acid, stearic acid, oleic acid, linoleic acid and linolenic acid. Fatty acid composition accounted for more than $60 \%$, and another grapefruit seed also contains a variety of physiological active ingredients, such as the class of bitter bitter, flavonoids, phospholipids, fat-soluble vitamins and other substances. China's grapefruit rich in resources, the use of waste grapefruit Xuan extract oil and to develop and use, cheap raw materials, you can get economic benefits, with a broad commercial production prospects.

Linoleic acid (LinoleicAcid) is a precursor of synthetic prostaglandins, is an important component of fat, linoleic acid deficiency for the maintenance of the normal function of membrane and oxidative acidification of the normal coupling will have an impact. Linoleic acid for high blood lipids, hypertension and cardiovascular and cerebrovascular diseases have a certain preventive effect. To prevent the deposition of serum cholesterol in human blood vessels. Have "blood vessels scavenger" reputation. Fan Ya and other studies have shown that linoleic acid on arteriosclerosis have a certain preventive effect. And can reduce the incidence of symptoms. Linoleic acid in vitro has a strong oxidation. Yandan and other linoleic acid on the different isomers of the physiological activity of the results show that linoleic acid has reduced the role of arterial wall toxicity. Effective prevention of atherosclerosis ${ }^{[2-4]}$. The effects of total linoleic acid on mice with type 2 diabetes mellitus were studied. The effects of total linoleic acid on the weight of mice were observed. The weight of the mice was lower than that of the untreated group. But also can reduce blood sugar, 
adjuvant treatment of diabetes. Zhang Wenbin studied the effect of linoleic acid on the body weight of obese rats. The weight of rats treated with linoleic acid decreased by $6.1 \%$. The reason is that linenic acid can change the expression of leptin and UCP2, to achieve the purpose of reducing weight. A large number of linoleic acid mainly affect the secretion of adipose tissue, so as to achieve the purpose of prevention of diabetes. However, studies have shown that large amounts of linoleic acid are also associated with low-density lipoprotein cholesterol (LDL-C), while high-density lipoprotein cholesterol (HDL-C) is reduced. Therefore, attention should be paid to the reasonable intake of unsaturated fatty acids In.

Flavonoids are widely distributed in plants. They are widely found in some plants and bales of nature. There are about 4 thousand species, and their molecular structure is different. Tang Qin et al. Studied the extraction of naringin from grapefruit skin and the anti-oxidative activity of naringin was determined by flow injection chemiluminescence assay. The naringin was placed at different times to determine the stability of naringin by measuring UV absorption. Tan Qingyun's research shows that the addition of naringin to toothpaste can play the purpose of anti-inflammatory, hemostasis $^{[5]}$. Zeng Liang found that adding naringin to pork can improve the color of pork to extend the shelf life. Jia Dongying and other preparation of pomelo peel if an iron complex can extend the shelf life of grease, but also can add trace elements. Huang Shirong and so on to grapefruit seed as raw material, ethanol as extractant, through single factor test and orthogonal test to determine the extraction of flavonoids in grapefruit seeds the best technology. The results showed that the extraction degree of flavonoids was as follows: the ratio of material to liquid> ethanol concentration> extraction time> extraction temperature ${ }^{[6]}$.

Grapefruit seed oil in Europe and the United States has been used as condiments, the use of micro-emulsification technology is the original domestic. There is hope to become a new technology to use grapefruit seed. Grapefruit seed oil contains a large number of unsaturated fatty acids, especially linoleic acid, as well as limonin, naringin and other active substances. With development value ${ }^{[7]}$. These substances in cell repair, anti-oxidation, anti-inflammatory, analgesic, cancer, antibacterial and so has some advantages. The pomelo seed oil can be made after the micro-milk to protect these substances to extend the shelf life. Can also be extracted from the ingredients of pomelo seed oil, development and utilization, made of drugs. Microemulsion preparation with its good transdermal, can also be made into cosmetics, so that grapefruit seed oil in the active ingredients into the skin better.

Chinese medicine, grapefruit sweet, sour, cold, there are stomach food, under the gas phlegm, light and so on color and other functions. Modern medicine research found that grapefruit meat contains a very rich vitamin $\mathrm{C}$ and insulin-like ingredients, it is hypoglycemic, hypolipidemic, weight loss, skin beauty and other effects ${ }^{[8]}$. Regular consumption, the high blood pressure, diabetes, vascular sclerosis and other diseases have adjuvant therapy, obese people have fitness Yang Yan function. Grapefruit also has a stomach, lungs, blood, bowel, and other benefits, can promote wound healing, sepsis, etc. have a good adjuvant effect. In addition, grapefruit contains physiologically active substances glucoside, can reduce blood viscosity, reduce the formation of thrombosis, so the cerebrovascular diseases, such as cerebral thrombosis, stroke, etc ${ }^{[9]}$. have a better preventive effect. The fresh grapefruit meat because of the composition of similar insulin, it is the ideal food for patients with diabetes.

\section{Materials, Reagents and Instruments}

Grapefruit seed, from the local supermarkets in the Shatian pomelo obtained, dried after the shell, and dried at $60{ }^{\circ} \mathrm{C}$ constant temperature, crushed, over 40 mesh sieve, spare; Rutin, China Pharmaceutical Group Shanghai biochemical reagents plant; petroleum ether, UV-9100 UV-Vis spectrophotometer, Beijing Ruili Analytical Instruments; BL-320H-type electronic balance, Japan Shimadzu company; electric blast temperature Drying oven, Shanghai South electric furnace oven factory; digital constant temperature water bath, Guohua Electric Co., Ltd. 


\section{Test Method}

Standard Curve Drawing. Accurately weighed $0.0150 \mathrm{~g}$ rutin standard, add the appropriate amount of $70 \%$ ethanol dissolved, constant volume to $100 \mathrm{ml}$ rutin standard solution. Respectively, the standard solution of the above standard $2.00,4.00,6.00,8.00,10.00 \mathrm{ml}$ in $25 \mathrm{ml}$ volumetric flask, add 1 $\mathrm{ml} 5 \% \mathrm{NaNO}_{2}$ solution, shake for $10 \mathrm{~min}$, then add $\left.1 \mathrm{ml} 10 \% \mathrm{~A} 1 \mathrm{NO}_{3}\right)_{3}$ solution, shake Equilibrated for $10 \mathrm{~min}$, and finally $10 \mathrm{ml}$ of $4 \% \mathrm{NaOH}$ solution was added and fixed to $25 \mathrm{ml}$ with $70 \%$ ethanol. After standing for $15 \mathrm{~min}$, the absorbance was measured at a wavelength of $510 \mathrm{~nm}$. Take the absorbance value $\mathrm{A}$ as the ordinate $(\mathrm{Y})$, the rutin mass concentration $(\mathrm{mg} / \mathrm{ml})$ as the abscissa $(\mathrm{X})$ to the rutin standard curve.

\section{Results and Discussion}

Orthogonal Test of Flavonoids Extracted from Grapefruit Seeds. On the basis of the above single factor test, orthogonal experiment was carried out by using L9 (34) orthogonal table, the ratio of material to liquid, ultrasonic power, extraction time and extraction temperature 4 factor 3 . The design and results of the orthogonal test are shown in Table 1.

Table 1 Design and results of orthogonal test

\begin{tabular}{cccccc}
\hline No. & temperature $\left({ }^{\circ} \mathrm{C}\right)$ & time $($ min) & $\begin{array}{c}\text { Material } \\
\text { liquid }(\mathrm{g} / \mathrm{mL})\end{array}$ & power(w) & $\begin{array}{c}\text { extraction rate } \\
(\mathrm{mg} / \mathrm{g})\end{array}$ \\
\hline 1 & 60 & 20 & $1: 30$ & 200 & 2.363 \\
2 & 60 & 25 & $1: 40$ & 240 & 2.668 \\
3 & 60 & 30 & $1: 50$ & 280 & 3.193 \\
4 & 65 & 20 & $1: 40$ & 280 & 2.895 \\
5 & 65 & 25 & $1: 50$ & 200 & 2.819 \\
6 & 65 & 30 & $1: 30$ & 240 & 2.582 \\
7 & 70 & 20 & $1: 50$ & 240 & 2.829 \\
8 & 70 & 25 & $1: 30$ & 280 & 2.551 \\
9 & 70 & 30 & $1: 40$ & 200 & 2.729 \\
average & 2.741 & 2.696 & 2.499 & 2.637 & \\
value 1 & & & & & \\
average & 2.765 & 2.679 & 2.764 & 2.693 & \\
value 2 & & & & & \\
average & 2.703 & 2.835 & 2.947 & 2.880 & \\
value 3 & & 0.156 & 0.448 & 0.243 & \\
Extreme & 0.062 & & & & \\
value & & & & & \\
\hline
\end{tabular}

The results showed that the primary and secondary effects of various factors on the extraction rate of flavonoids in grapefruit seed were the ratio of material to liquid> ultrasonic power> extraction time> extraction temperature. The optimum extraction conditions were as follows: extraction temperature was $65{ }^{\circ} \mathrm{C}$, ultrasonic power was $280 \mathrm{w}$, extraction time was $30 \mathrm{~min}$, and the 
ratio of material to liquid was 1:50 $(\mathrm{g} / \mathrm{mL})$. Under these conditions, the extraction rate of flavonoids from grapefruit seed was $3.380 \mathrm{mg} / \mathrm{g}$.

\section{Conclusion}

The results showed that the optimum extraction parameters were ultrasonic ratio $1: 50(\mathrm{~g} / \mathrm{mL})$, Ultrasonic power of $280 \mathrm{w}$, extraction temperature $65{ }^{\circ} \mathrm{C}$, extraction time $30 \mathrm{~min}$. Under these conditions, the extraction rate of flavonoids from grapefruit seed was $3.380 \mathrm{mg} / \mathrm{g}$. Ultrasonic enhanced extraction has the advantages of low temperature, high extraction efficiency and low cost, which can provide reference for industrial application.

\section{Acknowledgements}

This work is supported by Guangdong Province Student Innovation Experiment Project (No. 201710573029)

\section{References}

[1] Lin Z,Zhang J, Cui H, et al. Determination of aromatic amines in environmental water sample by hollow fiber-liquid phase microextraction and microemulsion electrokinetic chromatography^]. Journal of Chromatography A,2010,1217(26): 4507-4510.

[2] Hurtado-Fernandez E, Gomez-Romero M, Carrasco-Pancorbo A, et al. Application and potential of capillary electroseparation methods to determine antioxidant phenolic compounds from plant food materiaI[J]. Journal of Pharmaceutical and Biomedical Analysis, 2010,53(5): 1130-1160.

[3] Nguyen T, Do L, Sabatini D A. Biodiesel production via peanut oil extraction using diesel-based reverse-micellar microemulsions[J]. Fuel, 2010,89(9): 2285-2291.

[4] Song G, Lu C, Lin J. Application of surfactants and microemulsions to the extraction of pyrene and phenanthrene from soil with three different extraction methods[J]. Analytica Chimica Acta, 2007,596(2): 312-318.

[5] Ahmed E H,Raghavendra T, Madamwar D. An alkaline lipase from organic solvent tolerant Acinetobacter sp. EH28: Application for ethyl caprylate synthesis[J]. Bioresource Technology, 2010,101(10): 3628-3634.

[6] Pari a S. Surfactant-enhanced remediation of organic contaminated soil and water[J].Advances in Colloid and Interface Science, 2008,138(1): 24-58.

[7] Song G, Lu C, Lin J. Application of surfactants and microemulsions to the extraction of pyrene and phenanthrene from soil with three different extraction methods[J]. Analytica Chimica Acta, 2007,596(2): 312-318.

[8] Ouyang Y, Cho J S, Mansell R S. Simulated formation and flow of microemulsions during surfactant flushing of contaminated soil[J]. Water Research, 2002,36(1): 33-40.

[9] Childs J D, Acosta E, Knox R, et al. Improving the extraction of tetrachloroethylene from soil columns using surfactant gradient systems[J]. Journal of Contaminant Hydrology, 2004,71(1-4):27-45. 